\title{
The Role of Bis(p-Sulfonatophenyl)Phenylphosphine in Stabilizing Gold Nanoparticles
}

\author{
Ping Jin, Zhao Dai*, Tianrui Chang \\ School of Environmental and Chemical Engineering, \\ Tianjin Polytechnic University, \\ Tianjin, 300387, China \\ *daizhao@gmail.com
}

\begin{abstract}
This study investigated the role of bis( $p$-sulfonatophenyl)phenylphosphine (BSPP) in stabilizing gold nanoparticles in $0.5 \times$ TBE aqueous solution. Gold nanoparticles were made by the method of reduction of tetrachloroauric acid by sodium citrate, and then they were coated with BSPP. After centrifugation steps, the AuNPs/BSPP kept stable while the AuNPs aggregated without BSPP. When $\mathrm{NaCl}$ was added in the solution of AuNPs, with the presence of BSPP, AuNPs/BSPP was stable with little aggregation in a moderate concentration of $\mathrm{NaCl}$. The results showed that BSPP plays an important role in stabilizing gold nanoparticles.
\end{abstract}

Keywords-gold nanoparticle; BSPP; citrate; stabilization; aggregation

\section{INTRODUCTION}

Colloidal gold nanoparticles (AuNPs) have remained the focus of intensive research recent years[1]. AuNPs exhibit unique and distinctive physical and chemical properties different from bulk gold and gold atoms. These properties could be regulated by altering gold nanoparticles's size, shape, spacing and dielectric environment. Due to all the properties and increasingly preparation technology of controlled size and shape, AuNPs have found many successful applications in different fields as surface enhanced Raman scattering[2], catalysis[3], electronics[4], medical diagnosis[5] and biosensing[6,7]. The surface modification and protection of AuNPs from chemical corrosion are important requirements for many applications especially in biology. Unpurified AuNPs are relatively stable to be stored for a long time because of their surface charges. After purification, however, the AuNPs suspensions are susceptible to aggregation and easily affected by the environment. It may be necessary to find methods to modify the surface of gold nanoparticle to make it water-soluble, anti-cohesive, and affordable of complex physiological conditions [8].

Generally, AuNPs can be synthesized in the aqueous phase by the standard Turkerich method of reduction of tetrachloroauric acid by sodium citrate and be stabilized by citrate on their surfaces. However, the citrate group is a relatively weak ligand to AuNPs, which will result that the citrate groups can gradually detach from the particles' surfaces during the purification, such as washing and centrifugation steps. Therefore, AuNPs will quickly aggregate in the presence of salt. Many different researchs have been reported for the stabilization of AuNPs by modifying the surfaces of them with different ligands, such as DNA[9], protein[10,11], PEG[12], polyelectrolyte[13], self-assembly of functionalized thiols[14] to stabilize gold nanoparticles. However, the affinity between these ligands and AuNPs are too strong and the ligands cover the AuNPs surface firmly, which will lead to the difficulty for further applications.

In this paper, a simple method to stabilize gold nanoparticles in solution with bis(p-sulfonatophenyl)phenylphosphine (BSPP) were presented[15]. BSPP is a water-soluble triarylphosphine that forms coordination complexes with AuNPs. BSPP is a relatively stronger ligand which renders AuNPs a high stability in both water and ethanol solutions than citrate. It plays a role in increasing the tunnel barrier and making the colloids stable for months at a relatively moderate concentration. In addition, with BSPP protection, the salt dependent aggregation of AuNPs could be controlled and conjugation process with other biomoleculars could be done. In short, the modification of gold nanoparticles by BSPP is easy to achieve.

\section{EXPERIMENTAL}

\section{A. Materials}

Tetrachloroauric acid $\left(\mathrm{HAuCl}_{4}\right)$ and sodium citrate were purchased from WATSON Biotech. Tris(Hydroxymethyl)aminomethane, orthoboric acid and ethylene diamine tetraacetic acid (EDTA) were purchased from Alfa Aesar. bis(p-sulfonatophenyl)phenylphosphine dehydrate dipotassium salt (BSPP, 97\%) was purchased from Strem Chemicals. All chemicals were used without further purification. All solutions were prepared in deionized water from a Milli-Q water purification system.

\section{B. Synthesis of Gold Nanoparticles}

AuNPs were prepared by following procedures: $1 \mathrm{~mL}$ of $1 \%(\mathrm{w} / \mathrm{w}) \mathrm{HAuCl}_{4}$ aqueous solution was added to $100 \mathrm{~mL}$ of deionized water, when the solution reached sub-boiling state in magnetic stirring, $1 \mathrm{~mL}$ of $1 \%(\mathrm{w} / \mathrm{w})$ sodium citrate aqueous solution was added. Heating and stirring was continued till the solution boiled, kept the solution boiled for 30 minutes. When the solution cooled 
to room temperature, it was centrifuged and then restored in deionized water.

\section{Stabilization of AuNPs with BSPP}

The AuNPs solution was mixed with a certain amount of BSPP solution ( $50 \mathrm{mg} / \mathrm{mL}$ in water) in a microtube and incubated at $50{ }^{\circ} \mathrm{C}$ for 1 hour. The suspension was then centrifuged at $9000 \mathrm{r} / \mathrm{min}$ for 20 minutes, and the supernatant was discarded, the AuNPs at the tube bottom were resuspended in BSPP solution $(1 \mathrm{mg} / \mathrm{mL}$ in $0.5 \times \mathrm{TBE}$ ), and stored at $4{ }^{\circ} \mathrm{C}$. To make a comparison, another group of AuNPs suspension was centrifuged, and then resuspended in $0.5 \times \mathrm{TBE}$ buffer solution with no BSPP. The TBE buffer solution is widely used as biological buffer, the assembly of DNA[16] and protein to AuNPs is always carried out in this solution. The stability of BSPP to AuNPs was tested when $2 \mathrm{~mol} / \mathrm{L} \mathrm{NaCl}$ was added.

\section{RESULTS AND DISCUSSIONS}

\section{A. Stability of AuNPs with BSPP after Centrifugation} Steps

The solutions of AuNPs which were stabilized with citrate (AuNPs/citrate) and BSPP (AuNPs/BSPP) respectively were centrifuged twice at $10,000 \mathrm{r} / \mathrm{min}$ for 30 minutes. Figure 1 showed that the black spots appeared on the bottom of the tube of the AuNPs/citrate (a) suspension, which showed that the aggregation of AuNPs occurred, while the AuNPs/BSPP suspension (b) could be stable and resuspended in water after twice centrifugation. These results indicated that BSPP is a stronger ligand to stabilize AuNPs than citrate.

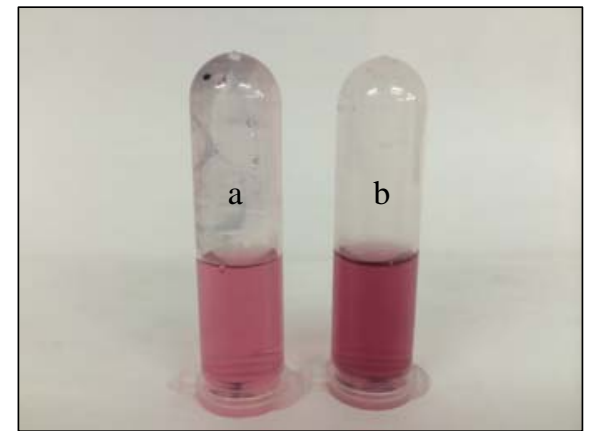

Figure 1. Photo of AuNPs suspensions after centrifugation steps, (a) with no BSPP; (b) stabilization with BSPP

\section{B. Stability of AuNPs with BSPP in Presence of $\mathrm{NaCl}$}

To further explore the performance of BSPP in stabilizing AuNPs, the aggregation behaviours in the presence of different concentrations of $\mathrm{NaCl}$ were carried out. A series of $\mathrm{NaCl}$ solutions with final concentrations raging $0,50 \mathrm{mmol} / \mathrm{L}$, and $100 \mathrm{mmol} / \mathrm{L}$ were injected into AuNPs/citrate and AuNPs/BSPP solutions, respectively, and the results were shown in Figure 2.

When BSPP existed, the colour of AuNPs/BSPP suspension faded slightly when the concentration of $\mathrm{NaCl}$ was increased (Figure 2A (a, b, c)) and the UV absorption peak of AuNPs/BSPP suspension also had a slight decrease (Figure 2B (a, b, c)), which indicated that the AuNPs had a slight aggregate. However, when there was no BSPP, the salt exhibited a strong influence on AuNPs suspension (Figure 2A (d, e, f). The colour of AuNPs suspension faded extremely, especially at high $\mathrm{NaCl}$ concentration (Figure 2A (e, f)). Moreover, the huge decrease of UV absorption of AuNPs suspension (Figuer 2B (e, f)) indicated that the free AuNPs in aqueous suspension was minor and major AuNPs was aggregated when no BSPP existed.

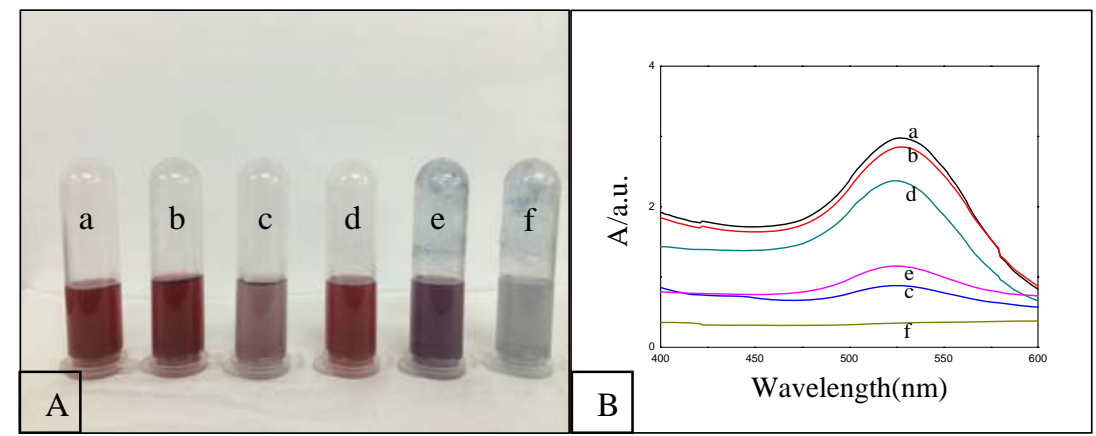

Figure 2. Photo (A) and UV-V absorption spectra (B) of AuNPs suspension, a,b,c were coated with BSPP, d,e,f were with no BSPP, the concentration of NaCl: (a,d): 0; (b,e): 50mM/L; (c,f): $100 \mathrm{mM} / \mathrm{L}$

Furthermore, The TEM of AuNPs suspensions were also used to investigate the BSPP role of stabilizing AuNPs (Figure 3). When there was no salt $(\mathrm{NaCl})$ adding into the AuNPs solutions, both the AuNPs/BSPP (Figure 3(a)) and AuNPs/citrate (Figure 3(d)) could keep stable and exhibit good dispensability; when the concentration of $\mathrm{NaCl}$ changed to $50 \mathrm{mmol} / \mathrm{L}$, AuNPs/BSPP (Figure 3(b)) dispersed well while AuNPs/citrate (Figure 3(e)) aggregated. When the concentration of $\mathrm{NaCl}$ was 100 $\mathrm{mmol} / \mathrm{L}$, AuNPs/BSPP (Figure 3(c)) began aggregation, but AuNPs/citrate (Figure 3(f)) aggregated extremely. 
These results indicated that BSPP was a relatively stronger ligand than citrate which rendered AuNPs a high stability in $0.5 \times \mathrm{TBE}$ solutions than citrate and it could protect AuNPs from aggregating with the presence of $\mathrm{NaCl}$.

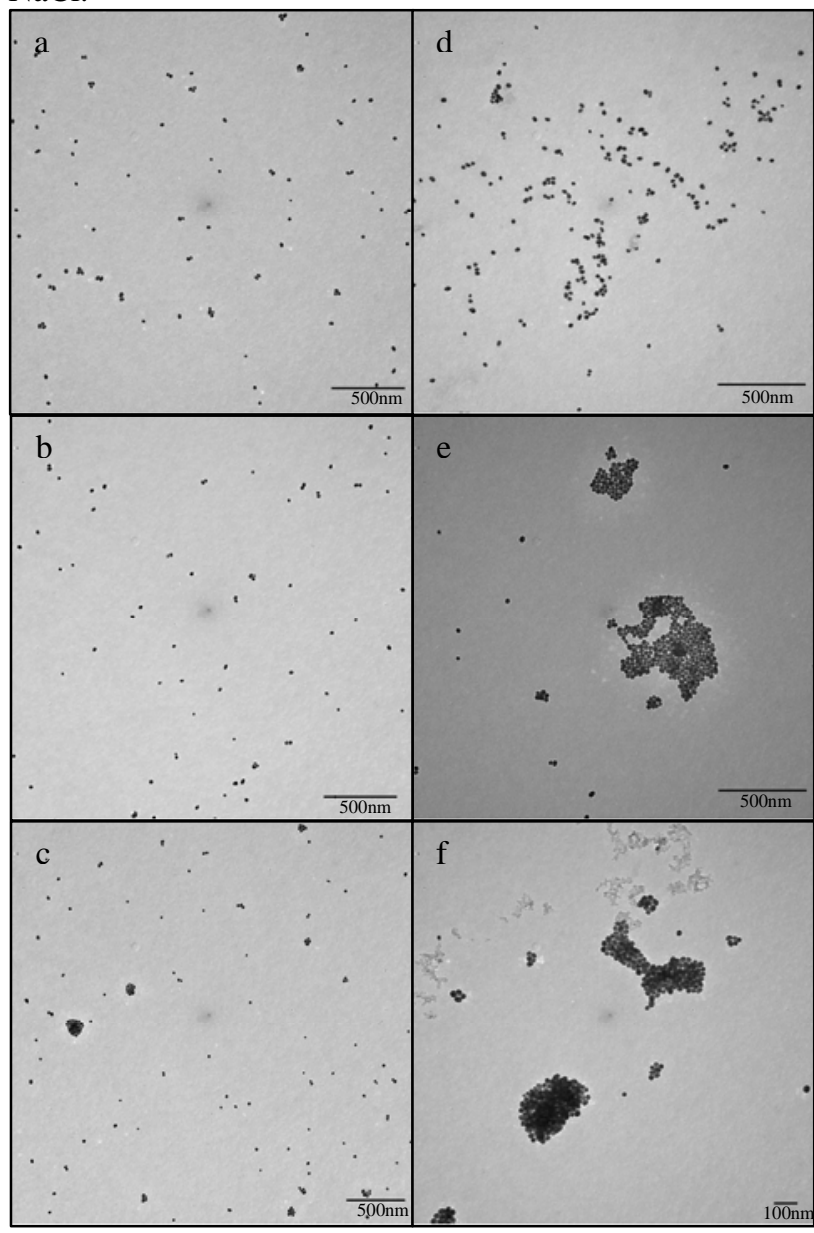

Figure 3. TEM images of AuNPs, a,b,c were coated with BSPP, d,e,f were with no BSPP, the concentration of $\mathrm{NaCl}$ : (a,d): 0 ; (b,e): $50 \mathrm{mM} / \mathrm{L}$; (c,f): $100 \mathrm{mM} / \mathrm{L}$

\section{CONCLUSIONS}

In this study, BSPP was proved as a relatively stronger ligand which renders AuNPs a high stability in $0.5 \times \mathrm{TBE}$ solutions than citrate. It plays a role in increasing the tunnel barrier and protects AuNPs from aggregation in the presence of a moderate salt concentration.

\section{ACKNOWLEDGEMENTS}

This work was supported by the National Natural Science Foundation of China (NO. 21172171).

\section{REFERENCES}

[1] Daniel, M.C. \& Astruc, D. Gold nanoparticles: assembly, supramolecular chemistry, quantum-size-related properties, and applications toward biology, catalysis, and nanotechnology. Chemical Reviews, 104(1), pp. 293-346,2004.

[2] Qian, X. Zhou, X. \& Nie, S. Surface-enhanced raman nanoparticle beacons based on bioconjugated gold nanovrystals and long range plasmonic coupling. Journal of the American Chemical Society, 130(45), pp. 14934-14935,2008.

[3] Zhou, K. \& Li, Y. Catalysis based on nanocrystals with well-defined facets. Angewandte Chemie International Edition, 51(3), pp. 602-613,2012.

[4] Moreira, H. Grisolia, J. Sangeetha, N.M. Decorde, N. Farcau, C. Viallet, B. Chen, K. Viau, G. \& Ressier, L. Electron transport in gold colloidal nanoparticle-based strain gauges. Nanotechnology, 24(9),2013.

[5] Qian, X.M. Peng, X.H. Ansari, D.O. Yin-Geon, Q. Chen, G.Z. Shin, D.M. Yang, L. Young, A.N. Wang, M.D. \& Nie, S.M. In vivo tumor targeting and spectroscopic detection with surface-enhanced Raman nanoparticle tags. Nature Biotechnology, 26(1), pp. 83-90,2008.

[6] Chen, J.I.L. Chen, Y. \& Ginger, D.S. Plasmonic nanoparticle dimers for optical sensing of DNA in complex media. Journal of the American Chemical Society, 132(28), pp. 9600-9601,2010.

[7] Thanh, N.T.K. Vernhet, A. \& Rosenzweig, Z. Gold nanoparticles in bioanalytical assays and sensors. Springer Series on Chemical Sensors and Biosensors, 3, pp. 261-277,2005.

[8] Guo, X. \& Ballauff, M. Spatial dimensions of colloidal polyelectrolyte brushes as determined by dynamic light scattering. Langmuir, 16(23), pp. 8719-8726,2000.

[9] Rosi, N.L. Giljohann, D.A. Thaxton, C.S. Lytton-Jean, A.K.R. Han, M.S. \& Mirkin, C.A. Oligonucleotide-modified gold nanoparticles for intracellular gene regulation. Science, 312(5776), pp. 1027-1030,2006.

[10] Xie, J.P. Zheng, Y.G. \& Ying, J.Y. Protein-directed synthesis of highly fluorescent gold nanoclusters. Journal of the American Chemical Society, 131(3), pp. 888-889,2009.

[11] Cheung-Lau, J.C. Liu, D. Pulsipher, K.W. Liu, W.R. \& Dmochowski, I.J. Engineering a well-orderd, functional protein-gold nanoparticle assembly. Journal of Inorganic Biochemistry, 130, pp. 59-68,2014.

[12] Lipka, J. S-Behnke, M. Sperling, R.A. Wenk, A. Takenaka, S. Schleh, C. Kissel, T. Parak, W.J. \& Kreyling, W.G. Biodistribution of PEG-modified nanoparticles following intratracheal instillation and intravenous injection. Biomaterials, 31(25), pp. 6574-6581,2010.

[13] Zikich, D. Borovok, N. Molotsky, T. \& Kotlyar, A. Synthesis and AFM charecterization of poly(dG)-poly(dC)-gold nanoparticle conjugates. Bioconjugate Chemistry, 21, pp. 544-547,2010.

[14] Woehrle, G.H. Brown, L.O. \& Hutchison, J.E. Thiol-functionalized, $1.5 \mathrm{~nm}$ gold nanoparticles through ligand exchange reactions: scope and mechanism of ligand exchange. Journal of the American Chemical Society, 127(7), pp. 2172-2183,2005.

[15] Han, X.G. Goebl, J. Lu, Z.D. \& Yin, Y.D. Role of salt in the spontaneous assembly of charged gold nanoparticles in ethanol. Langmuir, 27(9), pp. 5282-5289,2011.

[16] Bai, X. Wu, J.J. Han, X.G. \& Deng, Z.X. Probing DNA's interstrand orientation with gold nanoparticles. Analytical Chemistry, 83(13), pp. 5067-5072,2011. 